\title{
Sinking of light intruders in a shaken granular bed
}

\author{
Iván Sánchez, ${ }^{1, *}$ Gustavo Gutiérrez, ${ }^{2}$ Iker Zuriguel, ${ }^{3}$ and Diego Maza ${ }^{3}$ \\ ${ }^{1}$ Centro de Física, Instituto Venezolano de Investigaciones Científicas, Apartado Postal 89000, Caracas 1020-A, Venezuela \\ ${ }^{2}$ Departamento de Física, Universidad Simón Bolívar, Apartado Postal 1080, Caracas 1080-A, Venezuela \\ ${ }^{3}$ Departamento de Física y Matemática Aplicada, Universidad de Navarra, 31008 Pamplona, Spain
}

(Received 30 March 2010; published 23 June 2010)

\begin{abstract}
We present an experimental study of the displacement of a light intruder immersed in a vibrated granular bed. Using high speed video we resolve the motion, during one cycle of oscillation, of a cylindrical object inside a Plexiglas box partially filled with grains. We report experimental evidence that, in the absence of convection, at least two forces are behind the intruder's motion: an air drag force-due to the airflow through the granular bed-and a buoyancy force produced by an air-mediated granular fluid.
\end{abstract}

DOI: 10.1103/PhysRevE.81.062301

PACS number(s): 45.70.Mg

\section{INTRODUCTION}

Segregation occupies a privileged spot in the study of granular materials due to its importance in industrial processes. There is segregation in several configurations as rotating drums, hoppers, mixers, pipes, and in vertically vibrated media (for this last case, Kudrolli's review [1] is a good starting point). A simple problem that has been avidly studied is the segregation of a single object - or intrudermuch larger than the grains, immersed in a granular column. Most studies report the rise of this intruder when the column is shaken vertically. However, there are cases where the intruder sinks, specifically when the intruder density is lower than the bulk density of the granular material, and when the frequency of oscillation is around $10 \mathrm{~Hz}$ [2-6]. The sinking of light intruders has not been reported in the absence of air or in cases when interstitial air does not influence the dynamics of the granular medium in a significant way (i.e., grain diameter $>1 \mathrm{~mm}$ ).

Two mechanisms have been proposed to explain the sinking: the first one (by Möbius et al. [4,5]) was based on the air drag and the distinct effect that it has on the grains and the intruder. The other (by Gutiérrez et al. $[6,7]$ ) was based on a cyclic fluidization mediated by air. It has been shown that a pressure gradient develops during a single cycle of oscillation due to the separation of the granular column from the bottom of the container [8-12]. When the bed ascends, a low pressure region is created at the bottom and thus air flows down. When the bed descends, the pressure gradient reverses sign and air flows upward. Based on this airflow, Möbius et $a l$. assume an air drag force acting both on the granular column and the intruder. The acceleration of the intruder and the column due to this force ends up being proportional to the pressure gradient and inversely proportional to their respective densities. Hence, a light intruder will suffer a greater acceleration than that of the column and when the bed ascends, the light intruder will be pushed against the grains located underneath. If the intruder is below a certain critical height, it will be capable of displacing granular material and move downward with respect to the bed. On the contrary,

\footnotetext{
*ijsanche@ivic.gob.ve
}

when the bed descends, the light intruder will be pushed upward with respect to the bed. This mechanism captured many features of rising and sinking intruders (both heavy and light). The implementation of the model through numerical simulations, agreed quantitatively with experimental data of rising intruders, but could not offer quantitative information the sinking of light intruders without taking additional parameters into consideration [5].

Gutiérrez et al. [6,7] observed the sinking of a light intruder with density lower than that of the vibrated granular bed. They explain their experimental results in terms of an active buoyancy force and a reactive drag. These forces are assumed to arise from the granular fluid produced by the air flowing through the granular bed. Interestingly, both mechanisms are based on the role of the air. This seems reasonable as it has been experimentally shown that light intruders do not sink in vacuum [3,5], immersed in large grains [13], or shaken in recipients with the bottom permeable to air [7].

In order to shed light on the validity of the two mechanisms proposed for the sinking of light intruders and whether they coexist or not, in this work we approach this problem by minimizing the air drag on the intruder without altering the interaction of air with the granular bed. We used high speed video to register the displacement of a light intruder with respect to the bottom of the bed. From these measurements we obtain clear experimental evidences of at least two different mechanisms - which involve air-that should be considered in order to understand the motion of light intruders.

\section{EXPERIMENTAL SETUP}

Our experimental setup consisted of an intruder immersed in a granular bulk contained in a quasi-two-dimensional (quasi-2D) Plexiglas rectangular box $(280 \mathrm{~mm}$ tall, $200 \mathrm{~mm}$ wide, and $15 \mathrm{~mm}$ deep) (see Fig. 1). The box was mounted on an electromagnetic shaker driven by a function generator that supplied a sinusoidal excitation with $13 \mathrm{~Hz}$ of frequency and $5.9 \mathrm{~mm}$ of amplitude (implying a maximal adimensional acceleration $\Gamma=4.0)$. An amplifier was used to amplify the function generator signal. Experiments were done at atmospheric pressure and prior to each realization the inner walls were covered with anti-static spray. The granular material consisted on glass spheres of diameter between 180 and 


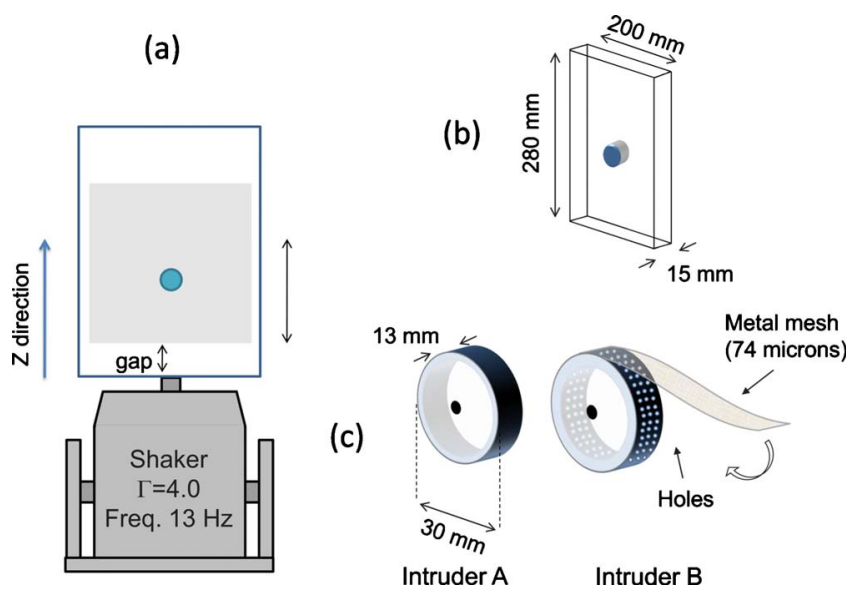

FIG. 1. (Color online) (a) Sketch of the experimental setup. (b) Container dimensions. (c) Description of intruder properties. Both intruders had the same exterior dimensions and the same density. Intruder $\mathbf{A}$ had a solid cylindrical wall. Intruder $\mathbf{B}$ had its cylindrical wall drilled regularly and covered with a metallic mesh, so as to reduce the air drag.

$300 \mu \mathrm{m}$. At rest, the granular column occupied two thirds of the total height with a porosity that was measured to be 0.44 , and a bulk density $\rho_{g}=1.4 \mathrm{~g} / \mathrm{cm}^{3}$.

We used two cylindrical intruders (named $\mathbf{A}$ and $\mathbf{B}$ ) with diameter $30 \mathrm{~mm}$ and height $13 \mathrm{~mm}$, allowing enough space between the circular faces of the intruders and the vessel walls to hold $\sim 10$ grains. This is a large enough quantity of grains to avoid the blockage of the intruder (a common problem in Hele-Shaw geometries), but small enough to allow intruder visualization. Both intruders had the same density $\rho_{i}$ such that $\rho_{i} / \rho_{g} \approx 0.4$. The only difference between both intruders was that the lateral wall of intruder $\mathbf{A}$ was solid whereas intruder $\mathbf{B}$ had its wall drilled regularly and covered with a metallic mesh in order to make it permeable to air but not to the grains [see Fig. 1(c)].

All the experiments started by placing the center of the intruder $60 \mathrm{~mm}$ above the vessel floor. Then, the vibration was switched on and the whole vessel area was recorded with a fast camera at one megapixel resolution and 3000 frames per second. This particular configuration was chosen to maximize the speed and resolution while maintaining a large enough field of view to monitor the movement of the intruder during more than 10 cycles of vibration. The absence of convection was checked with an intruder with relative density $\rho_{i} / \rho_{g} \approx 1$. After hundreds of cycles, this intruder did not show a net displacement within our experimental resolution. Thus, if there is a contribution of convection to the motion of the intruder, it will not be significant in a single cycle of oscillation.

\section{RESULTS}

In Fig. 2 we show the vertical position of two intruders as a function of the period of oscillation $T$ along ten cycles of oscillation. Additionally, the vertical position of the granular column is presented. The results of the intruders movement in the container's reference frame (red triangles) reveal that
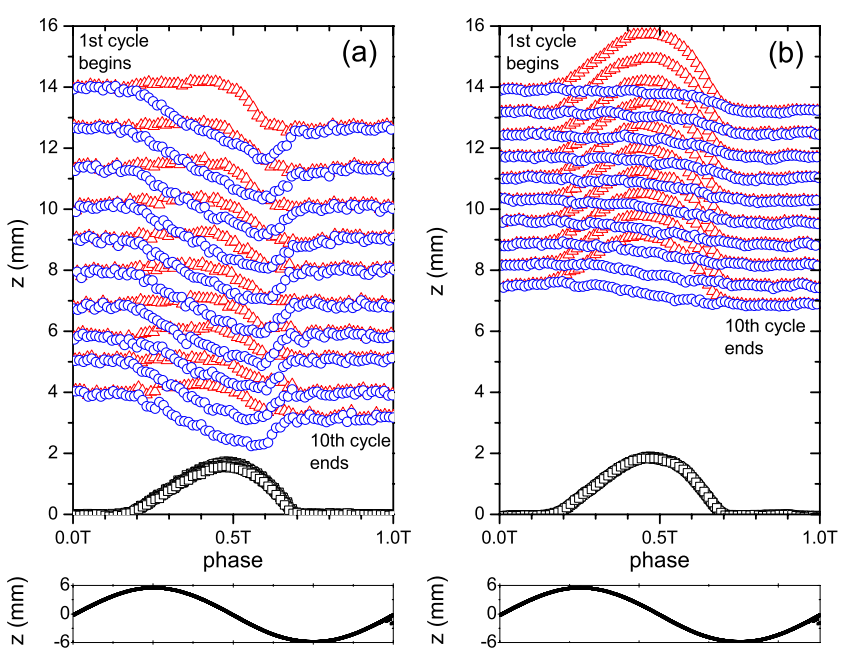

FIG. 2. (Color online) Vertical position $(z)$ of intruders tracked during ten cycles as a function of the period of oscillation $T$. The symbols represent the intruder position with respect to the container (red triangles), the intruder position with respect to the bottom of the granular column (blue circles) and the position of the bottom of the column (black squares). The position of the container is shown at the bottom. (a) Intruder A. (b) Intruder B.

both perform a net downward motion in each cycle. After ten cycles of vibration, intruder $\mathbf{A}(\mathbf{B})$ moves $10.9 \pm 0.2 \mathrm{~mm}$ $(7.1 \pm 0.2 \mathrm{~mm})$, for an average speed of $14.2 \pm 0.4 \mathrm{~mm} / \mathrm{s}$ $(9.2 \pm 0.3 \mathrm{~mm} / \mathrm{s})$. Hence, the porous intruder sinks with a speed that is $64 \%$ that of the intruder without holes. Apart from this quantitative difference in the sinking speed, it is important to note that there is a qualitative difference in the displacement of the intruders during a single cycle. When the granular column detaches from the bottom at around $0.15 \mathrm{~T}$, the impermeable intruder (A) seems to keep a constant position with respect to the container. Then at $0.5 \mathrm{~T}$, just when the column starts to descend, the intruder moves downward with respect to the container. The porous intruder (B) ascends (descends) in the frame of reference of the container when the granular column ascends (descends).

Once this different behavior has been observed for both intruders, we further investigate the movement in a single cycle by presenting the intruder's movement with respect to the granular column (blue circles in Fig. 2). This has been carried out by subtracting the position of the granular column to the displacement in the vessel's reference frame. Among the several ways that can be used to estimate the position of the granular column we chose to take the bottom free surface as a reference (black squares in Fig. 2). The reason for this lies in the fact that the bottom surface is clearly defined and it is easy to detect with the camera. The results of the intruders' movement with respect to the granular column prove that the impermeable intruder moves during the whole time in which the granular material is detached from the bottom and rests steady during the rest of the cycle. The same behavior is found for the permeable intruder although the mobility is strongly reduced and it is necessary to make a closer look. This is nicely observed in Fig. 3, where the average displacement over ten cycles is presented for both intruders.

The results of Fig. 3 reveal that the movement of intruder A is correlated with the air flow through the column during 

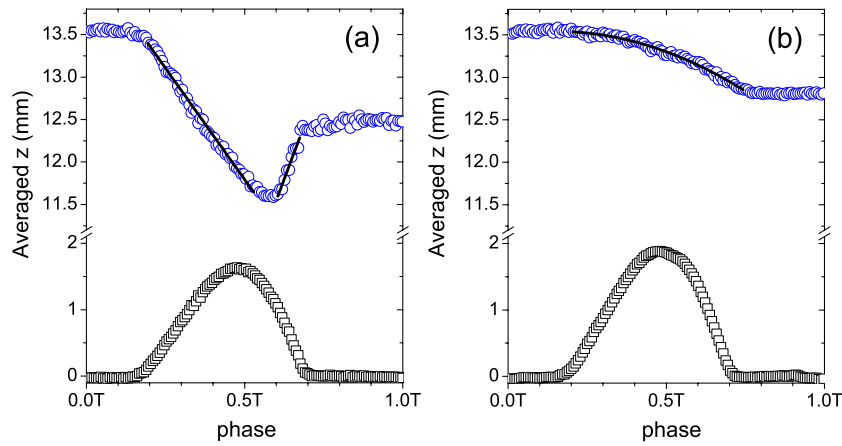

FIG. 3. (Color online) Average displacement of intruders tracked during ten cycles with respect to the granular column (blue circles). The averaged gap is also shown (black squares). (a) Intruder $\mathbf{A}$ sinks and rises with a roughly linear dependence on time. The downward fit has a slope of $-66.6 \pm 0.5 \mathrm{~mm} / \mathrm{s}$ and a correlation coefficient $R^{2}=0.99201$. The upward fit has a slope of $116 \pm 1 \mathrm{~mm} / \mathrm{s}$ and correlation coefficient $R^{2}=0.9896$. (b) Intruder B descends monotonically describing a parabola. The parabolic fit gives a quadratic coefficient of $-320 \pm 10 \mathrm{~mm} / \mathrm{s}^{2}$, a linear coefficient $-6.6 \pm 0.8 \mathrm{~mm} / \mathrm{s}$ and an independent term $13.53 \pm 0.01 \mathrm{~mm}$, with correlation coefficient $R^{2}=0.98936$.

the whole cycle. The intruder starts moving when the gap starts developing. While the gap is increasing there is a downward airflow which drags the intruder, who performs a roughly linear motion with speed $66.5 \pm 0.5 \mathrm{~mm} / \mathrm{s}$. After the gap reaches its maximum at $0.5 \mathrm{~T}$ the airflow reverses its sign and shortly afterward (around $0.55 \mathrm{~T}$ ) the intruder does the same, rising a little with speed $116 \pm 4 \mathrm{~mm} / \mathrm{s}$. Instead, intruder $\mathbf{B}$ is not affected by the direction of the airflow as it sinks monotonically. The moving part of the trajectory is well fitted by a parabola, suggesting a motion which is constantly accelerated at $640 \pm 2 \mathrm{~mm} / \mathrm{s}^{2}$.

Finally, the results of the position of the granular bottom displayed in Fig. 3 reveal that the porosity of the intruder has some effect on the movement of the granular column during a single cycle of vibration. The average gap obtained with intruder $\mathbf{B}$ reaches a maximum height that is $15 \%$ higher than the obtained with intruder $\mathbf{A}$. This is reasonable if we think that the presence of the porous intruder acts as an air passage along the granular column and therefore, the effective porosity increases.

\section{DISCUSSION}

In the previous section, we described the movement of sinking intruders during a single cycle of oscillation and analyzed the main differences caused by a modification of the permeability of the intruder. Let us now discuss the implications of these results and the relationship with the mechanisms proposed in the literature for light intruder sinking (air drag and air-mediated buoyancy). If we consider the close relation between the trajectory of the intruder $\mathbf{A}$ and the airflow through the granular column, it is logical to propose the air drag as the mechanism behind intruder motion. However, the motion of intruder $\mathbf{B}$ evidences that an additional ingredient is necessary to fully explain the motion. Due to the holes on its cylindrical wall, air drag is less effective on intruder $\mathbf{B}$ than on intruder $\mathbf{A}$. Then, it may be thought that this is the reason for which the average sinking velocity of the intruder $\mathbf{B}$ is smaller than that of intruder $\mathbf{A}$. However, if air drag on intruder $\mathbf{B}$ were simply lower but comparable to the drag on intruder $\mathbf{A}$, one would expect their trajectories to be qualitatively similar. Clearly, the results presented in this manuscript reveal that this is not the case suggesting that other mechanism, apart from the air drag force, is behind the sinking of porous intruders.

From Fig. 3(b) we can conclude that the motion of intruder $\mathbf{B}$ is not correlated with the airflow through the column and hence, it cannot be explained by assuming an air drag force. This is clear if we observe its motion at $0.6 \mathrm{~T}$. At this point the air changes its direction of flow but this does not cause any observable change in the displacement of the intruder B. The permeable intruder displays a smooth accelerated downward motion that suffers no qualitative change during the time that the gap is different from zero (from 0.15 to $0.7 \mathrm{~T}$ ). During this time the airflow fluidizes the granular material and then, it is reasonable to think that a buoyancy force can arise $[6,14]$. Note that, in this case, the fluidization does not depend on the direction of the air flow. While the bed is in a fluidized state, the effective gravity, seen from the reference frame of the granular bed, points upward. Consequently, a buoyancy force felt by the light intruder must point downward (in the same way that a light intruder suffers an upward buoyancy force when the gravity points downward). In this work we give experimental evidence that the sinking of a light intruder in a vibrated granular column can be attributed to two different air-mediated mechanisms. If the intruder is impermeable to the air, the motion is clearly driven by an air drag force, because it responds to changes in the direction of airflow during a cycle of oscillation. If the intruder is permeable, no evidence of drag force is detected, and the sinking velocity is reduced in $36 \%$. In this case, the experimental results are consistent with the existence of a buoyancy force arising from an air-mediated fluidization of the granular bed. Consequently, a model that aims to quantitatively predict the motion of a light intruder in a granular bed should consider a quantification of both, an air drag force and a buoyancy force.

\section{ACKNOWLEDGMENTS}

This work was supported by Projects No. FIS2008-06034C02-01 (MICINN, Spain), PIUNA (University of Navarra) and No. IVIC-857 (IVIC, Venezuela). 
[1] A. Kudrolli, Rep. Prog. Phys. 67, 209 (2004).

[2] T. Shinbrot and F. J. Muzzio, Phys. Rev. Lett. 81, 4365 (1998).

[3] X. Yan, Q. Shi, M. Hou, K. Lu, and C. K. Chan, Phys. Rev. Lett. 91, 014302 (2003).

[4] M. E. Möbius, X. Cheng, G. S. Karczmar, S. R. Nagel, and H. M. Jaeger, Phys. Rev. Lett. 93, 198001 (2004).

[5] M. E. Möbius, X. Cheng, P. Eshuis, G. S. Karczmar, S. R. Nagel, and H. M. Jaeger, Phys. Rev. E 72, 011304 (2005).

[6] G. Gutiérrez, O. Pozo, L. Reyes, R. Paredes, J. Drake, and E. Ott, EPL 67, 369 (2004).

[7] L. Reyes, I. Sánchez, and G. Gutiérrez, Physica A 358, 466 (2005).
[8] R. Gutman, Trans. Inst. Chem. Eng. 54, 174 (1976).

[9] T. Akiyama and T. Naito, Chem. Eng. Sci. 42, 1305 (1987).

[10] B. Thomas and A. M. Squires, Phys. Rev. Lett. 81, 574 (1998).

[11] B. Thomas, M. Mason, and A. Squires, Powder Technol. 111, 34 (2000)

[12] J. M. Pastor, D. Maza, I. Zuriguel, A. Garcimartín, and J.-F. Boudet, Physica D 232, 128 (2007).

[13] D. A. Huerta and J. C. Ruiz-Suárez, Phys. Rev. Lett. 92, 114301 (2004).

[14] D. A. Huerta, V. Sosa, M. C. Vargas, and J. C. Ruiz-Suarez, Phys. Rev. E 72, 031307 (2005). 